Review

\title{
Oncogenes Orchestrate Immunosuppressive Stroma in Gastric Adenocarcinoma
}

Jiankang Jin, Shumei Song ${ }^{*}$, Jaffer A. Ajani *

Department of Gastrointestinal Medical Oncology, The University of Texas MD Anderson Cancer Center, Houston, TX 77030, USA; E-Mails: jjin@mdanderson.org; ssong@mdanderson.org; jajani@mdanderson.org

* Correspondence: Shumei Song and Jaffer A. Ajani; E-Mails: ssong@mdanderson.org; jajani@mdanderson.org

Academic Editor: Tapan K Bera

Special Issue: Molecular Cancer Therapeutics

OBM Genetics

2020, volume 4, issue 3

doi:10.21926/obm.genet.2003116
Received: June 30, 2020

Accepted: September 03, 2020

Published: September 16, 2020

\begin{abstract}
Gastric adenocarcinoma (GAC) is among the three most common cancers in the world. The majority of GAC patients are diagnosed in an advanced stage and have a median survival of $\sim 9$ months. There are limited effective therapeutic strategies available in the clinic and currently U.S. Food and Drug Administration (FDA) approved immune therapy is programmed death-1 (PD-1) antibodies (e.g. pembrolizumab) but only a few patients seem to benefit. Transformation to cancer occurs when multiple genes and cellular pathways are dysregulated in multi-cellular organisms. Mounting evidence supports that oncogenes orchestrate tumor immune suppressive stroma to foster tumor favoring microenvironmental niche. Thus, deeper understanding of the immunosuppressive mechanisms in tumor stroma especially orchestrated by notable oncogenes can allow exploration of novel avenues that may have an impact on patient outcome. In this review, we summarize current progress of notable oncogenes and pathways including Ras/Myc, EGFR/HER2, PI3K/mTOR, Wnt/ $\beta$-catenin, and Hippo/YAP pathways focusing on the interplay between these oncogenic pathways and
\end{abstract}

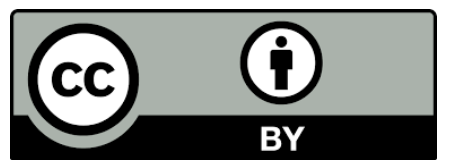

(C) 2020 by the author. This is an open access article distributed under the conditions of the Creative Commons by Attribution License, which permits unrestricted use, distribution, and reproduction in any medium or format, provided the original work is correctly cited. 
immunosuppressive stroma. Future potential novel targets and immune checkpoint blockage are discussed.

\section{Keywords}

Gastric adenocarcinoma; oncogenes; immune cells; immunosuppression; stroma; TME

\section{Introduction}

Cancer occurs, in multicellular organisms, when multiple oncogenes are activated along with simultaneous or sequential loss of function of tumor suppressor genes leading to multiple cellular pathways being deregulated. This process is driven by accumulated genetic abnormalities. According to the World Health Organization (WHO), cancer is the second leading cause of death globally and is a generic term for a large group of diseases that can affect any part of the body, other terms are malignant tumors and neoplasms. Gastric adenocarcinoma (GAC) is among the 3 most common causes of cancer deaths in the world (https://who.int/cancer). An oncogene literally is a cancer-causing gene, which is often mutated (gain-of-function mutation) or highly amplified. The normal functional proto-oncogenes are involved in the regulation of cell growth and proliferation, differentiation, and inhibition of apoptosis. Mutated proto-oncogenes become activated by a variety of genetic mechanisms including insertional/deletional mutagenesis, amplification, point mutations, and chromosomal translocations [1]. Here we summarize current research progress of selected notable oncogenes in GAC including Ras/Myc, epidermal growth factor receptor (EGFR)/HER2, PI3K/mTOR, Wnt/ $\beta$-catenin, and Hippo/YAP1.

\section{Notable Oncogenes in Cancers}

\subsection{Oncogenes Activated by Viruses}

A comprehensive book [2] in 2017 detailed all the major discoveries of viruses associated in human cancers from 1911 till now. Oncogene Myc (also as c-myc or MYC) was first identified in an avian myelocytoma virus and the other Ras gene was first identified in rat sarcoma virus long before they became associated with human malignancies [3]. The first confirmed oncogene SRC (protooncogene C-SRC; a non-receptor tyrosine kinase) was a homolog of previous avian sarcoma virus vSRC gene and transmitted by a chicken retrovirus Rous sarcoma virus (RSV) that acted as an oncogene upon infection [4], even though human genome has the RSV homolog in place. Table 1 briefly lists viral infections turned on oncogenes or mutated tumor suppressors, leading to cancer, some of them are marked as milestones in cancer molecular biology.

For GAC, The Cancer Genome Atlas (TCGA) analysis identified one of four molecular subtypes of GAC is Epstein-Barr virus+ (EBV+) tumors, in which detected PIK3CA mutations, CDKN2A silencing, JAK2 amplification and programmed death-ligand-1 (PD-L1) and PD-L2 amplifications [5]. 
Table 1 Viruses, activated host oncogenes, deregulated tumor suppressors and associated cancers

\begin{tabular}{|c|c|c|c|c|}
\hline Viruses & Host Oncogenes /Tumor suppressors & Cancers & Notes & References \\
\hline Rat sarcoma virus & Activated Ras & Rat sarcoma & & {$[3,4]$} \\
\hline Epstein-Barr virus (EBV) & $\begin{array}{l}\text { Sstr1 methylated; } \mathrm{Bcl}-2 \text { overexpression; viral miR- } \\
\text { Bart3-3p induces Ras }{ }^{\mathrm{G} 2 \mathrm{~V}} \text {, PIK3CA mutations }\end{array}$ & Gastric Cancer & $\begin{array}{l}\text { One of four molecular } \\
\text { subtypes of GAC }\end{array}$ & {$[6-9]$} \\
\hline Human herpesvirus 8 (HHV-8) & $\begin{array}{l}\text { Induced FAK and SRC; Induced ERK1/2, NFKB and } \\
\text { NRF2 }\end{array}$ & Kaposi's sarcoma & & {$[10,11]$} \\
\hline Avian myelocytoma virus & Activated Myc & Avian myelocytoma & & {$[3,4]$} \\
\hline Epstein-Barr virus (EBV) & Myc translocation and activation & Burkitt lymphoma & $\begin{array}{l}\text { First human tumor virus in } \\
1964\end{array}$ & [12] \\
\hline Rous sarcoma virus (RSV) & Transforming SRC & Chicken Rous sarcoma & $\begin{array}{l}\text { First discovered oncogene } \\
\text { transmitted by a virus }\end{array}$ & {$[2,4]$} \\
\hline Epstein-Barr virus (EBV) & NFKB activation and/or activated PI3K/mTOR/AKT & Nasopharyngeal carcinoma (NPC) & & [13] \\
\hline Hepatitis B virus (HBV) & $\begin{array}{l}\text { HBV-human fusion transcript IncRNA (HBx) binds \& } \\
\text { impairs p53 }\end{array}$ & Hepatocellular carcinoma & Oncogenic IncRNA & {$[14]$} \\
\hline Human papillomavirus (HPV) & Viral E6 destabilizes p53; viral E7 degrades pRB & $\begin{array}{l}\text { Cancer in cervix, vagina, and vulva } \\
\text { and oropharyngeal, anal, penile, } \\
\text { and cutaneous carcinomas. } \\
\text { Tumors in rabbits }\end{array}$ & & {$[2]$} \\
\hline Human T-cell leukemia virus type 1 (HTLV-1) & Viral Tax gene activates/modifies NFkB/Rel family & Adult T-cell leukemia (ATL) & First retrovirus discovered & {$[2,15]$} \\
\hline Lentiviruses (HIV, SIV) & $\begin{array}{l}\text { Vpr gene product arrests human cells in } \mathrm{G}_{2} \text {. Viral } \\
\text { integrase hijacks LEDGF/p75 }\end{array}$ & Human HIV & & {$[16,17]$} \\
\hline Polyoma virus and simian virus 40 (SV40) & $\begin{array}{l}\text { Viral proteins bind p53 and pRB; Loss-of function p53 } \\
\text { and pRB }\end{array}$ & Murine polyoma & & {$[4,18]$} \\
\hline
\end{tabular}




\subsection{Oncogenes Activated by Bacteria}

Bacteria are normally not considered as agents that cause cancers, but research has found more connections. Common bacteria Staphylococci pneumoniae infection was reported to be associated with leukemia, Iymphoma, or myeloma; Staphylococci bovis infection was speculated to be associated with colorectal cancer in a case study in 1951; Salmonella typhimurium was reported to be associated with gallbladder cancer, etc. [19]. Persistent Mycoplasma penetrans infection in immunodeficient mice demonstrated lower expression of tumor suppressors p53 and p21 and higher expression of oncogenes HRas, nuclear factor-KB (NFKB-p65) and B-cell lymphoma 2 (Bcl-2), which are associated malignant transformation [20].

For GAC, a book chapter detailed bacterial infections causing cancers, such as Helicobactor pylori causes GAC and mucosa-associated lymphoid tissue (MALT) lymphoma [19]. H. pylori is the most important risk factor for GAC, which is found in $90 \%$ of GAC (especially in Asia). This bacterium has 3 virulence factors: cytotoxin-associated gene A (CagA), CagA pathogenicity island (Cag PAI) and vacuolating cytotoxin $A(\operatorname{Vac} A)[5,21]$. In gastric epithelium, oncogenic Cag $A$ activates oncogenes $\beta$ catenin, MET (also as c-Met or cMet), EGFR, PI3K and AKT, results in loss of function of tumor suppressor genes RUNX1, CDH1 (E-cadherin) and TFF1, and suppresses the immune milieu by recruiting tumor-associated macrophages [TAMs] and alter the microRNA (miRNA) profile [5]. $H$. pylori is the most important risk factor and carcinogen for GAC. Long-term infection leads to chronic atrophic gastritis and pre-cancerous changes [6].

H. pylori virulence factors CagA and VacA activate multiple pathways including Ras/Myc, MAPK, $N F K B$, activating protein (AP), Wnt/ $\beta$-catenin, PI3K in stomach epithelial cells into production of inflammatory cytokines, such as IL-1 $\beta$, IL-6, IL-8, necrosis factor alpha (TNF $\alpha$ ), INF- $\gamma$ I and MCP1, resulting in an inflammatory response, mucosal damage and eventually gastric carcinogenesis [22].

\subsection{Tumorigenesis Sustained by Activated Oncogene and Dysregulated Protooncogenes}

A single mutated or dysregulated oncogene (a gene fusion) can lead to cancer but that is not common. In most cancers, multiple oncogenes drive cells in the context of loss-of-function tumor suppressor genes. Such a complex process can take years before a fit clone emerges as established cancer. In GACs, promoter CpG island hypermethylation (CIMP) is frequently found, high levels of CIMP are associated with poor prognosis in younger patients and are associated with mutated oncogenes $\beta$-catenin, HER2, KRas, and PIK3CA [5].

In the transgenic mouse models with inducible oncogenes under the control of regulated promoters, oncogenes are turned on and tumors can emerge, extinction of expression often leads to rapid disappearance of the tumor, and those genes with tumor maintenance include KRas, HRas, Myc, Neu (HER2), Wnt, EGFR, fusion gene Bcr-Abl (breakpoint cluster region-Tyrosine-protein kinase Abl1), and MET, etc. [23]. Oncogene MDM-2 is overexpressed as much as 100 -fold in as many as one-third of human sarcomas [16]. The first identified human oncogene was by R. Weinberg in 1982 and later was identified as a mutated allele of HRas [24]. Myc proto-oncogene coordinates genes essential to cellular programs required for normal as well as neoplastic cellular growth and proliferation. With Myc inactivation, cancer cells undergo proliferation arrest, which induces immune activation, angiogenesis arrest, cell senescence, and apoptosis [25]. 


\section{Interplay Between Oncogenes and the Immunosuppressive Stroma in the Tumor Microenvironment (TME)}

\subsection{Innate, Adaptive Immune Cells and Immunomarkers}

A comprehensive review of immunology in GAC peritoneal carcinomatosis (PC) was recently reviewed by our group [26]. Macrophages (M $\phi)$, neutrophils, dendritic cells (DCs), and natural killer (NK) cells are part of innate immunity and directly defend against invading pathogens and cancer cells and convene adaptive immune systems by $T$ and $B$ lymphocytes. Innate immunity, adaptive immunity, cytokines, chemokines, immune checkpoints proteins are parts of cell immunity. Cytokines are regulators of the innate and adaptive immune systems, which control proliferation, differentiation, effector functions, and survival of leukocytes [27]. Chemokines are small, secreted proteins/peptides being the largest subfamily of cytokines that mediate immune cell trafficking and lymphoid tissue development. In TME, chemokines can be expressed and produced by tumor cells and other cells including immune cells and stromal cells [28]. For tumor proliferation, cancer cells upregulate vascular endothelial growth factor (VEGF), MMPs, immunosuppressive IL-10, transforming growth factor-beta (TGF- $\beta$ ), and epithelial-mesenchymal transition (EMT). For metastases, cancer cells downregulate NK ligands to become disseminated tumor cells (DTCs), recruit immunosuppressive macrophages, and increasing neutrophil extracellular traps (NETs). Notable oncogenes can orchestrate an interplay between tumor cells and immune suppressive stroma as stated below.

\subsection{Ras and Myc}

KRas (also known as K-ras, KRAS) is a small GTPase that mediates downstream signaling from growth factor receptors which binds guanosine triphosphate and diphosphate nucleotides. KRas is the most frequently mutated oncogene. The mutated and activated KRas binds and activates kinases sequentially in an axis of KRas-RAF1/BRAF/ARAF-MEK1/MEK2-ERK1/ERK2/transcription factors ELK1/C-JUN, leading to cell proliferation [29]. In pancreatic cancer, mutated KRas, together with mutated tumor suppressor CDKN2A (P16), TP53 and SMAD4 accounted for more than 90\% of tumors [30]. Pancreatic intraepithelial neoplasia (PanIN) grading of 4 types are based on KRas, and loss-of-function CDKN2A, TP53, BRCA1 and 2 [29]. In GAC, KRas has a relatively low mutation/amplification frequency in the range of 7-20\% [31]. Myc was first identified in an avian myelocytoma virus $\mathrm{v}$-myc long before it becomes associated with human malignancies [3]. Myc is a family of transcription factors, including the human gene c-myc (Myc), I-myc (MYCL), and n-myc (MYCN). In cancer, Myc is often persistently expressed, leading to the expression of many other oncogenes, which are involved in cell proliferation, contributing to the formation of cancer [32].

\subsubsection{Interplay Between Ras/Myc and Other Oncogenic Signaling.}

In GAC transformation, Mycoplasma penetrans infection in immunodeficient mice showed lower expression of tumor suppressors p53 and p21 and higher expression of oncogenes HRas (also as transforming protein p21), NFKB-p65 subunit and $\mathrm{Bcl}-2$ indicating that coordination among oncogenes in malignant transformation [20]. In five GAC cell lines, MET activation as well as mutations in KRas and $\mathrm{CDH} 1$ was associated with cetuximab resistance [33]. By administration of 
rapamycin and CPT-11, embryonic stem cell-expressed Ras (ERas) was upregulated in GAC cells, which induces a cascade of oncogenes of AKT, mTOR, and the nuclear factor (NFKB) in conferring chemoresistance [34]. These studies indicate that oncogenes EGFR, MET, and KRas, AKT, NFKB interplay and lead to resistance to therapies. Tanshinone IIA (Tan-IIA) extracted from an herb inhibits GAC AGS cells by decreasing VEGFR, HER2, Ras, RAF, MEK, and ERK expression [35]. An interplay between Ras/Myc, other oncogenes and their elements of immunosuppression is illustrated in Figure $1 \mathrm{~A}$.

\subsubsection{Interplay Between Ras/Myc and the Immune Microenvironments}

The first direct evidence of KRas mediated inflammation and immune microenvironments reported by Okumura et al. (2010) that conditional expression of oncogenic KRas mutation in K19expressing putative gastric epithelial progenitor cells induced gastric atrophy, metaplasia, and dysplasia by activating inflammatory pathways and displaying recruitment of BMDCs (bone marrowderived cells) to the TME. Most importantly, they found that oncogenic KRas mutation in gastric epithelial cells upregulated cytokine and chemokine expression including IL-1, IL-6, CXCL1, and CXCL5 which create a tumor favoring TME that facilitates tumorigenesis of GAC [36]. In a mouse lung model of KRas ${ }^{G 12 D}$-driven adenomas, co-activation of Myc drives the immediate transition to highly proliferative and invasive adenocarcinomas marked by highly inflammatory, angiogenic, and immune-suppressed stroma. Epithelial-derived CCL9 and IL-23 were identified as the principal instructing signals and orchestrate recruitment of macrophages, angiogenesis, and PD-L1dependent expulsion of $T$ and $B$ cells for a tumor immune suppressive environments [37]. Casey et al. (2016) reported that Myc regulates the expression of two immune checkpoint proteins on the tumor cell surface: the innate immune regulator CD47 (cluster of differentiation 47) and the adaptive immune checkpoint PD-L1. Suppression of Myc in mouse tumors and human tumor cells reduced levels of CD47 and PD-L1 expression. Myc inactivation in mouse tumors down-regulated CD47, PD-L1 expression, and enhanced the antitumor immune response [38].

In GAC, PD-L1 expression was associated with loss-of-function ARID1A mutation. The study found in $3 \mathrm{MSI}-\mathrm{H}$ (MSI-high, microsatellite instability-high) tumors showing highest expression of PD-L1 had simultaneous KRas mutation and loss of ARID1A. Loss of ARID1A triggers upregulation of PD-L1 through PI3K/AKT signaling pathway. KRas mutation boosts PD-L1 expression in MSI-H GACs, suggesting a possible synergistic role boosting PD-L1 [39].

\subsection{EGFR/HER2}

The epidermal growth factor receptor (EGFR) family is composed of four closely-related members: ErbB-1 (HER1 or EGFR), ErbB-2 (HER2, or c-erbB-2), ErbB-3 (HER3), and ErbB-4 (HER4), all of which play a critical role in regulating cell growth, proliferation, and migration of tumor cells. GACs overexpress this gene family in a heterogeneous pattern, especially EGFR and HER2. Improved survival from the use of trastuzumab (Herceptin) has paved the way for ErbB receptor familytargeted treatments in GAC [40]. Among GAC patients, 15\% show HER2 overexpression and/or amplification, and this percentage increases somewhat in gastroesophageal junction (GEJ) adenocarcinomas [41]. 


\subsubsection{EGFR/HER2 and Mechanisms of Target Resistance}

Study of EGFR-directed antibody cetuximab in GAC cell lines revealed that high EGFR expression and low levels of receptor activation were associated with cetuximab responsiveness, however, MET activation and mutations of $\mathrm{KRas}$ and $\mathrm{CDH} 1$ was associated with cetuximab resistance [33]. In one study, evaluation of HER2 status in 97 GAC patients showed that HER2 3+ (highest) expression are likely to benefit the most from trastuzumab [42]. Two GAC cell lines with HER2 amplification, one sensitive ( $\mathrm{NCl}-\mathrm{N} 87$ ) and other one insensitive (MKN-7) to trastuzumab, inhibition of the mTOR/S6K using everolimus was the key in enhancing fluorouracil-induced apoptosis specifically in GAC cells. mTOR inhibitors may be attractive alternative drugs in GACs with HER2 amplification/over-expression [43]. Inhibition of checkpoint kinase 1 (Chk1) phosphorylation enhanced HER2-targeting lapatinib sensitivity of HER2-positive MKN-7 cells, which was shown by potentiated anti-proliferative effect [44].

Maron and Catenacci (2017) listed oncogene HER2, VEGFR2, EGFR, MET, and FGFR2 (fibroblast growth factor type 2) for targeted therapies for esophagogastric cancer (only Her2 and FGFR2 may remain useful targets) [45]. Cancers that overexpress EGFR, HER2, and HER3 are uniquely sensitive to agents that disrupt HER2 and EGFR protein folding. Disruption of disulfide bond formation by disulfide disrupting agents (DDAs) kill HER2/EGFR overexpressing cells. Interference with proline isomerization in HER2/EGFR overexpressing cells also induces cancer cell death. The peptidyl-prolyl isomerase inhibitor cyclosporine A (CSA) selectively kills EGFR+ or HER2+ breast cancer cells in vitro by activating caspase-dependent apoptotic pathways. Further, CSA synergizes with the DDA to kill HER2+ tumor cells in vivo, which may apply to EGFR/ HER2 overexpressed esophageal and GACs [46]. Tanshinone IIA (Tan-IIA) extracted from a herb inhibits GAC AGS cells by upregulating expression of PARP and caspase-3 but decreased VEGFR, HER2, Ras, Raf, MEK, and ERK, suggesting all those oncogenes together facilitate the proliferation and survival of cancers [35].

\subsubsection{Interplay Between EGFR/Her2 and the Immunosuppressive TME}

Through tumor-derived exosomes (TEXs), cancer cells are able to transfer activated EGFR to host macrophages and thereby suppress innate antiviral immunity assessed by measuring interferon- $\beta$ (IFN $\beta$ ). EGFR is required for TEX-mediated immunosuppression, kinase MEKK2 is identified in macrophages as an effector of TEX-delivered EGFR that negatively regulates the antiviral immune responses [47]. Macrophage inhibitory cytokine-1 (MIC-1) in the TME can inhibit the secretion of TNF- $\alpha$, reducing tumor killing of macrophages [48]. MIC-1 induced transactivation of HER2 in breast cancer and GAC cells, and induced significant phosphorylation of AKT and ERK-1/2. siRNA-mediated downregulation of HER2 significantly reduced not only phosphorylation of AKT and ERK-1/2 but also invasiveness of cells induced by MIC-1. MIC- 1 induced expression of HIF- $1 \alpha$ and its target VEGF, via the activation of the mTOR signaling. Thus, MIC-1 may participate in the malignant progression of cancer cells that overexpress HER2 [49].

Trastuzumab has been the mainstay of therapy for HER2+ breast cancer and GAC through antibody-dependent cellular phagocytosis (ADCP) $(50,51)$. It was unexpectedly realized that after ADCP macrophages inhibited NK cell-mediated antibody-dependent cellular cytotoxicity (ADCC) and T cell-mediated cytotoxicity in breast cancers and lymphomas. Combined treatment with anti-HER2 antibody and inhibitors of PD-L1 and IDO (indoleamine 2,3-dioxygenase) enhanced antitumor immunity and anti-HER2 therapeutic efficacy in the mouse models. Trastuzumab therapy has 
significantly upregulated PD-L1 and IDO in the TAMs of HER2+ breast cancer samples, correlating with poor trastuzumab response. It is unveiled a deleterious role of ADCP macrophages in cancer immunosuppression and suggested that oncogene-directed antibody plus immune checkpoint blockade may provide synergistic effects against cancer [51].

It was reported that the endosomal sorting complexes required for transport (ESCRT)-associated protein ALIX as a regulator of both EGFR activity and PD-L1 surface presentation in basal-like breast cancer (BLBC) cells. ALIX-deficient tumors were larger and showed an increased immunosuppressive environment by induced EGFR activity and redistribution of PDL-1 to the cell surface [52]. VEGF-, HER2- and EGFR-targeted agents are sometimes used to treat gastric, esophageal, and colorectal cancers. However, treatment outcomes are poor in most gastrointestinal (GI) cancers, antibodies such as targeting immune checkpoints anti- PD-1 (nivolumab and pembrolizumab) and PD-L1 (atezolizumab) therapies have not done well in general [53]. Interplay between oncogenes EGFR/HER2 and their immunosuppression is illustrated in Figure $1 \mathrm{~B}$.

\subsection{PI3K/mTOR}

The PI3K/mTOR pathway is an intracellular signaling pathway important in regulating the cell cycle involving AKT and PI3K which phosphorylates and activates their downstream signaling. The mammalian target of rapamycin (mTOR), a mechanistic target of rapamycin, is a kinase, which has core component of two distinct protein complexes, mTOR complex 1 (mTORC1) and mTOR complex 2 (mTORC2) which regulate different cellular processes. mTOR regulates cell growth, cell proliferation, cell motility, cell survival, protein synthesis, autophagy, and transcription.

\subsubsection{PI3K/mTOR and Target Strategies}

As previously mentioned, activation of PI3K/mTOR mediated resistance of trastuzumab in GAC cell lines with HER2 amplification/over-expression. Thus, inhibition of mTOR/S6K using everolimus may be attractive alternative [43]. In gastroenteropancreatic neuroendocrine neoplasms (GEPNENs), PKI-587 is a highly potent, novel dual inhibitor of PI3K and mTORC1/C2, with dose-dependent inhibition of proliferation. In all cell lines treated with PKI-587, all led to cell cycle arrest and induction of apoptosis and successfully suppressed activity of mTORC1 target 4E-BP1, a crucial factor for tumorigenesis. PI3K/mTOR dual targeting showed promising new therapeutic approach in neuroendocrine tumor [54].

Inhibition of mTORC1 with mTOR inhibitor rapamycin may lead to an induction of AKT phosphorylation in cancer cells via mTORC2 activation paradoxically. A study found that rapamycin additionally up-regulated both insulin-like growth factor 1 receptor (IGF-IR) and HER2 expression. mTORC2 inhibition reduced the phosphorylation of GSK-3 and NFKB, and significantly impaired cancer cell motility. Inhibition of mTORC2 may abrogate unfavorable signaling effects of mTOR inhibitors [55]. Rapamycin-insensitive companion of mTOR (RICTOR) amplification defines a subset of advanced GAC and is sensitive to AZD2014-mediated mTORC1/2 inhibition. Treatment of RICTOR amplified patient-derived cells with selective drugs targeting AKT, mTORC1/2 demonstrated preferential sensitivity to the mTORC1/2 inhibitor (AZD2014). Knockdown of RICTOR reversed patient-derived cells' sensitivity to AZD2014, validating the importance of RICTOR amplification in solid tumors including GAC [56]. Activation of IGF-IR signaling is implicated in tumor cell mobility of various cancers, including GAC [57]. 


\subsubsection{Interplay Between PI3K/mTOR and the Immunosuppressive TME}

Tumor associated macrophages (TAMs) are the main regulatory cell type in the TME. The fatty acid enriched tumor environment itself was sufficient to induce the regulatory phenotype of TAMs, including the up - regulation of classical markers like CD206, IL - 6, VEGF $\alpha$, MMP9 or Arg1. mTORC2 activation played a critical role in the generation of the suppressive myeloid cell phenotype. Cellspecific inhibition of DGAT1 and 2 prevented oleate-induced polarization into immunosuppressive TAMs [58]. GAC mesenchymal stem cells (MSCs) derived IL-8 induced PD-L1 expression in GAC cells via STAT3/mTOR-Myc signal axis. MSCs exerted broad immunosuppressive potential, modulating the activity of cells either in the innate or adaptive immune system to promote tumor progression. MSCs enhanced PD-L1 expression in GAC cells resulting in resistance of GAC cells to CD8+ T cells cytotoxicity. The study proved that IL-8 derived from MSCs induced PD-L1 expression in GAC cells via Myc regulated by STAT3 and mTOR signaling pathways [59].

Chemokine CXCL12 is a small protein that functions in normal hematopoietic stem cell homing in addition to repair of damaged tissues through its receptor CXCR4. CXCR4 plays an important role in metastatic progression and destination. CXCL12 activation induced phosphorylation of AKT, and then phosphorylated mTOR (pS6K and 4E-BP1) in disseminated GAC cells [60]. CXCL12/CXCR4 activated the PI3K/AKT/mTOR pathway in GAC line MKN-45. Rapamycin inhibited secretion/expression of CXCL12/CXCR4. The mTOR pathway played an important role in CXCL12/CXCR4-mediated cell migration, and drugs targeting the mTOR pathway can be used for therapy of GAC expressing high levels of CXCL12 [61]. Binding of CXCL12 to CXCR4 led to activation $\mathrm{P} 13 \mathrm{~K} / \mathrm{mTOR}$ and MEK/ERK while binding to CXCR7 leads to $\beta$-arrestin mediated signaling, leading to anti-apoptotic signaling $\mathrm{BCl}-2$ and survivin upregulation, and promoting the EMT. A CXCR4 antagonist AMD3100 (plerixafor or Mozobil), is the most frequently used drug targeting the CXCL12CXCR4/CXCR7 axis for GI cancers. Thus, the CXCL12-CXCR4/CXCR7 axis acts as a mechanism of immune resistance in GI cancers. [62]. Gl cancers are frequently associated with chronic inflammation and excessive secretion of IL-6 family cytokines, which promote tumorigenesis through persistent activation of the GP130/JAK/STAT3 pathway. A parallel pathway that activated excessive mTORC1 alongside STAT3 in human intestinal-type GACs. Activation of GP130-dependent mTORC1 is required for inflammation-associated Gl tumorigenesis [63]. The interplay between $\mathrm{PI3K} / \mathrm{mTOR}$ signaling and TME is shown in Figure 1C. 


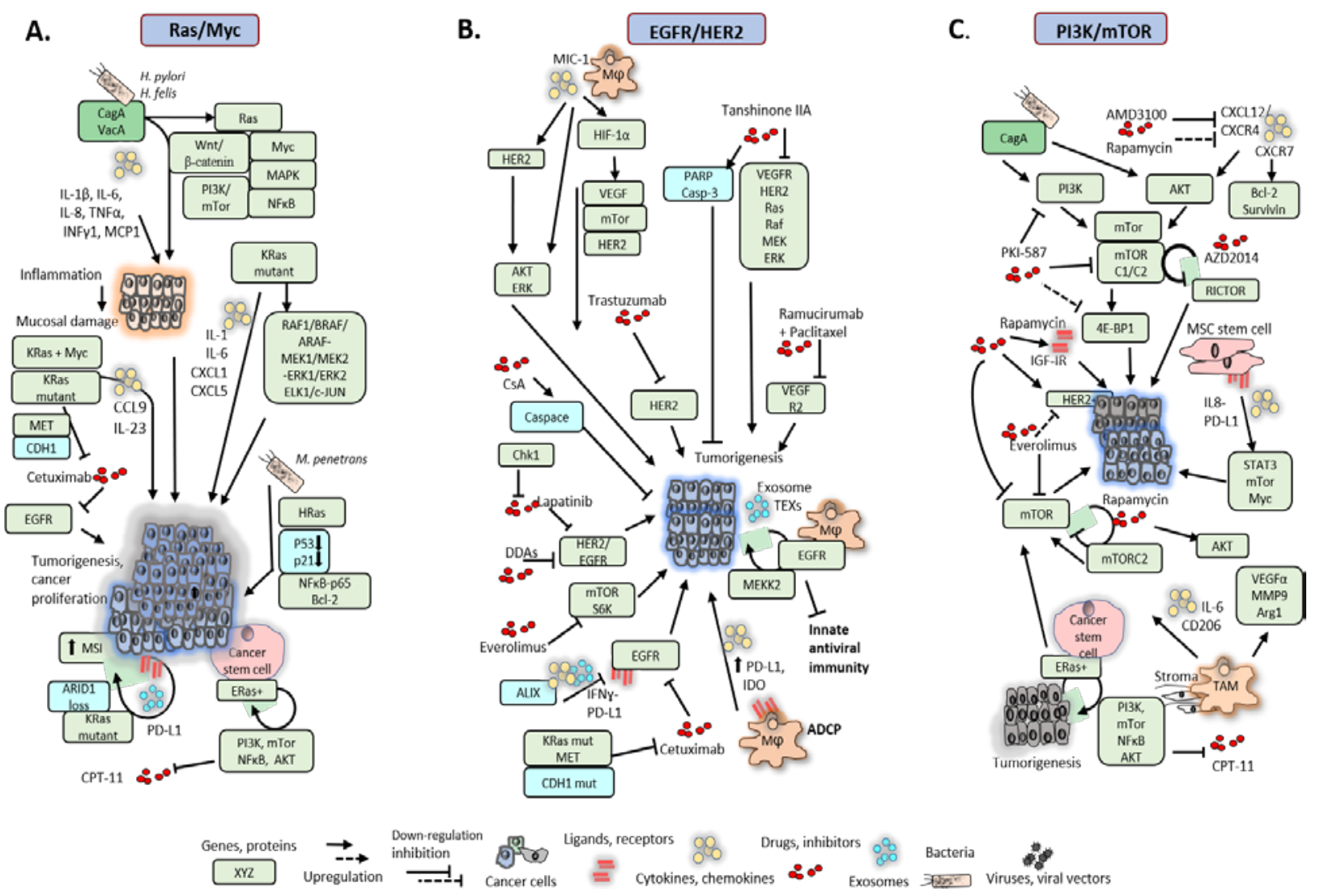

Figure 1 Interplay between oncogenes and their immunosuppressive TME. A. Ras/Myc mediated interplay. Bacterial virulence factors CagA/VacA turn on a plethora of oncogenes Ras, Wnt/ $\beta$-catenin, Myc, NFKB, PI3K/mTOR, MAPK, elicit multiple proinflammatory cytokines IL-1 $\beta$, IL-6, IL-8, TNF $\alpha$, INF 1 1, MCP1 to cause epithelial mucosa inflammation and mucosal damage, with persistent bacterial infection and oncogenic KRas and Myc driving and cytokine CCL9, IL-23 secretion, tumorigenesis and cancer proliferation proceed. Other factors, such as cancer stem cell associated ERas, tumorassociated PD-L1 with help of mutated ARID1 and KRas and MSI-H, all drive GC tumorigenesis and proliferation. B. EGFR/HER2 mediated interplay. Tumor-associated M2 M $\phi$ secreted cytokine MIC-1 and turned on many oncogenes, HER2, HIF1, VEGF, mTOR, AKT and ERK, to cause tumorigenesis. By ADCP, M2 M induced cell checkpoint ligand PD-L1/IDO and secrets IL-1 $\beta$ that results in cancer immunosuppression. Exosome TEXs recruit M2 M $\phi$ and turn on EGFR/MEEK2 to promote tumor cells self-renewal, tumorigenesis and proliferation, suppressing innate antiviral immunity. C. PI3K/mTOR pathways: bacterial virulence factor CagA turned on PI3K and AKT. PI3K and its direct target mTOR (including components $\mathrm{mTORC1/2}$ ) interact a host of other oncogenes STAT3, Myc, VEGFa, HER2, MMP9, Arg1, NFkB, AKT to promote gastric tumorigenesis and proliferation. AKT was proved to regulate mTOR. Second, rapamycin inhibits mTOR, thus suppresses gastric cancer progression, but rapamycin induces IGF-IR and HER2 paradoxically, impairs cancer cell motility. Third, gastric cancer MSC stem cells-derived IL-8 inducing PD-L1 modulates the activity of cells either in innate or adaptive immune system to promote tumor progress. ERas expressed in human GC exert chemoresistance. TAMs in tumor stroma upregulated many oncogenes. 


\subsection{Wnt/B-catenin}

Wnt proteins are cysteine-rich glycoproteins that bind to the extracellular domain of frizzled receptor and lipoprotein receptor-related protein. Wnt/ $\beta$-catenin signaling is an evolutionarily conserved pathway that is known to be involved in embryonic development and tissue homeostasis. An aberrant activation of this pathway with accumulation of $\beta$-catenin, a core protein, in the nucleus promotes the transcription of many oncogenes [64].

\subsubsection{Wnt/ $\beta$-catenin and Oncogenic Interplay}

In GAC, two signaling pathways, Wnt/ $\beta$-catenin and NFKB, are dysregulated in $70 \%$ of GAC patients. H. pylori's virulence factor CagA interacts with E-cadherin, leading to $\beta$-catenin accumulation in cytoplasm and nucleus. The overexpression of Wnt2 is correlated with cytoplasmic/nuclear $\beta$-catenin accumulation in GAC in Chinese patients. The expression of Wnt2 was positively correlated with lymph node metastases [6]. Activation of $\mathrm{Wnt} / \beta$-catenin signaling has been important in $\mathrm{Gl}$ tumorigenesis. It has been suggested that the promotion of Wnt/ $\beta$-catenin activity beyond the threshold is important for carcinogenesis [65]. As core protein, nuclear $\beta$-catenin in Wnt pathway activates many oncogenes such as Myc and CyclinD1 [64]. Figure 2A illustrates the interplay among $\mathrm{Wnt} / \beta$-catenin signaling, other oncogenes, and related immunosuppressive stroma.

\subsubsection{Interplay Between Wnt/ $\beta$-catenin and the Immunosuppressive Stroma}

Macrophages promote $\mathrm{Wnt} / \beta$-catenin activity in gastric tumorigenesis in mouse stomach and GAC cells [65]. Macrophage-derived TNF $\alpha$ activates Wnt/ $\beta$-Catenin during $\mathrm{Gl}$ cancer development, mutated tumor suppressor APC (APC $\triangle 716)$ sustains Wnt/ $\beta$-catenin expression. With other factors such as $H$. pylori infection, macrophages produce TNF $\alpha$, promotes nuclear accumulation of $\beta$ catenin via AKT and GSK3 $\beta$ in neoplastic epithelial cells. This process can be exasperated by production of Wnt ligands by stromal cells that bind frizzled receptors, leading to inhibition of the $\beta$-catenin degradation complex (APC, AXIN, GSK3b) [66]. A drug lupeol does not exhibit toxicity to normal cells and tissues but was noted to inhibit the proliferation of GAC cells in a dose-dependent manner and induced proliferation of NK cells in GAC cell lines. The possible mechanism is that lupeol might increase expression of PFP, IFN $\gamma$, and CD107a via activation of PI3K/AKT and Wnt/ $\beta$-catenin signaling [67]. In a study, $\beta$-catenin demonstrated its immunosuppressive role in GAC through a $\beta$ catenin-CCL28-Treg cell axis, clarifying an important mechanism for immunosuppression. Expression of $\beta$-catenin directly activated its target CCL28 which recruited Tregs. In a clinically relevant mouse GC model established by bacterium $\mathrm{H}$. felis infection and $\mathrm{N}$-methyl- $\mathrm{N}$-nitrosourea (MNU) treatment, inhibition of $\beta$-catenin/TCF activity suppressed CCL28 expression and Treg cell infiltration in the stomach, and anti-CCL28 antibody attenuated Treg cell infiltration and tumor progression. Diphtheria toxin-induced Treg cell ablation restrained GAC progression in mice, clarifying the tumor-promoting role of Tregs [68].

Early studies proved TNF $\alpha$ as an immune regulator in GAC. One study revealed that TNF $\alpha$ induced up-regulation of Wnt10A in GAC cell lines in the same fashion as H. pylori [69]; Wnt10B is upregulated by TNF $\alpha$ in human GAC cell line MKN45 [70]; Expression and regulation of Wnt5A by TNF $\alpha$ in MKN45 Cells was reported [71]. H. pylori's CagA dysregulates and activates Wnt/ $\beta$-catenin [6], the active CagA activates multiple pathways including $W \mathrm{nt} / \beta$-catenin among others in stomach 
epithelial cells into production of inflammatory cytokines, such as IL-1 $\beta$, IL-6, IL-8, TNF $\alpha$, INF- $\gamma$ I and MCP-1 [22]. A mouse study used steroid analog for osteoporosis, bazedoxifene, to suppress GP130dependent tumor growth of the GI epithelium, where tumors arose through excessive GP130/STAT3 signaling in response to cytokine IL-11 [72]. Using conditionally replicating adenoviral vectors (CRAds) which integrated an adenoviral E1A gene and apoptosis-inducing gene IL-24 inhibited proliferation and tumor growth in mouse GAC xenografts, which were with $\beta$-catenin mutation status or aberrant Wnt signaling [73]. IL-24 is unusual in that a cytokine exhibits tumor suppressor property.

Bone morphogenetic protein and activin membrane-bound inhibitor (BAMBI) has been confirmed as a transmembrane glycoprotein and is a member of immune checkpoint TGF- $\beta$ family. Knockdown of BAMBI in aggressive GAC cell lines significantly inhibited cell invasion and proliferation. $\beta$-catenin expression was downregulated as a result of BAMBI knockdown, and TGF- $\beta$ was downregulated in a similar manner [74]. TGF- $\beta 1$ works with immunity Treg to promote cancer stem marker leucine-rich repeat containing $G$ protein-coupled receptor 5 (Lgr5), leading to Wnt/ $\beta$ catenin upregulation in GAC [75].

\subsubsection{Wnt/ $\beta$-catenin, Cancer Stem Cells (CSC) and Immunosuppression}

$H$. pylori's VacA induces Wnt/ $\beta$-catenin signaling, promotes CSC properties in GAC cells and cell proliferating [6]. Cancer cell stemness together with oncogenic Wnt/ $\beta$-catenin expression sustained GAC progression and drug resistance. SALL4, an embryonic stem cell marker, has a direct interaction with Wnt signaling, its overexpression is correlated with lymph node metastases in GAC [6]. A study demonstrated expression of transglutaminase-1 (TGM1) was upregulated in GAC, and that TGM1 expression levels were correlated with patient survival, which promotes stemness and chemoresistance in GAC cells by regulating Wnt/ $\beta$-catenin signaling [76]. In another study, Lgr5 was examined in 180 GACs by immunohistochemistry (IHC), and in 80 pairs of GACs for analysis of Th1/Th2 (T helper cells) cytokines. Lgr5 expression was up-regulated in GAC cells co-cultured with Tregs or treated with exogenous TGF- $\beta 1$. This up-regulation was partially inhibited by the TGF- $\beta 1$ neutralizing antibody, or TGF- $\beta 1$ receptor antagonist. $\beta$-catenin was up-regulated with high Lgr5 expression induced by exogenous TGF- $\beta 1$, and this up-regulation was inhibited by TGF- $\beta 1$ receptor antagonist. Thus, Tregs and TGF- $\beta 1$ promoted CSC marker Lgr5, led to Wnt/ $\beta$-catenin upregulation, all interplayed and conferred poor prognosis [75].

By generating mouse GAC cell line and comparing its metastatic variant, stem cells antigen-1 (Sca-1) was identified as a cell surface marker, which was mostly upregulated in metastatic variant, Sca-1-high mouse GAC cells demonstrated increased tumorigenicity. Sca-1 expression was downregulated by TGF- $\beta$ pathway activation and $W n t / \beta$-catenin pathway inhibition. A chromatin immunoprecipitation (ChIP) analysis demonstrated that Sca-1 was a $\beta$-catenin/LEF1 target gene. Sca-1-high allografts were more resistant to cisplatin/fluorouracil chemotherapy and overexpressed $\mathrm{Bcl}-\mathrm{xL}$. Sca-1 is a novel CSC enrichment marker that mediates TGF- $\beta$ and $W n t / \beta$-catenin signaling [77].

A study proved that human GAC cells subvert gene expression and cytokine production by reprogramming of "naïve" MSCs into specialized tumor associated MSC equipped with a tumorpromoting phenotype for the MSC-mediated support of cancer stemness in GAC. CSC properties are sustained in vivo through an interplay between GAC and tumor associated MSC by activating the R- 
spondin/Lgr5 axis and Wnt/ $\beta$-catenin signaling pathway. $\beta$-Catenin + cell clusters show $\beta$-catenin nuclear localization, indicating the activation of the $\mathrm{Wnt} / \beta$-catenin signaling pathway in these cells [78].

\subsection{Hippo/YAP1}

The Hippo pathway and its coactivator YAP1 is an essential pathway that regulates cell proliferation, apoptosis, organ growth, and homeostasis of the Gl tissues. This pathway is highly conserved in mammals [79]. Dysregulation of Hippo pathway is associated with initiation, development, and distant metastases [6, 80-84]. YAP1 is frequently elevated in a number of cancer types such as lung, colorectal, ovarian, liver, and prostate cancers, where it acts as a powerful tumor promoter for tumor progression [85].

\subsubsection{Hippo/YAP1 and Oncogenic Interplay}

An early study by using integrative genomic analyses of Wnt5A revealed that Snail, CD44, G3BP2 and YAP1 are Wnt5A target genes [86]. Fat4 functions as a Hippo signaling regulator, loss of Fat4 due to gene mutation was detected in a variety of tumors including GAC, thus Fat 4 was recognized as a tumor suppressor. By silencing Fat4 using shRNA in GAC cells, Fat4 suppression led to the increase in phosphorylated YAP1 ( $p$-YAP) and YAP1 nuclear accumulation. Transfection of a fulllength Fat4 downregulated $\mathrm{p}$-YAP1 and inhibition of the cell cycle progression. Intriguingly, Fat4 reduction also leads to accumulation of cytoplasmic $\beta$-catenin. Fat4-silenced cells treated with 5-FU, cisplatin, oxaliplatin and paclitaxel individually demonstrated less sensitivities to these drugs. IHC analysis revealed that Fat4 expression was significantly reduced in GAC tissues [87]. Large tumor suppressor 1 (LATS1) being part of the Hippo pathway plays important role for cellular homeostasis. Loss of LATS1 promotes growth and metastases of GAC cells through YAP1 upregulation. Overexpression of LATS1 decreased GAC cell proliferation and invasion in vitro and inhibited tumor growth and liver metastases in mice; depletion of LATS1 expression restored the invasive phenotype. The Hippo/YAP pathway was required for LATS1-induced inhibition of cell growth and invasion, and LATS1 abated nuclear transfer of YAP1, downregulated YAP1, PCNA, CTGF, MMP-2, MMP-9, Bcl-2 and CyclinD1 expression and upregulated $\mathrm{p}$-YAP and Bax expression. The finding proved that LATS1 is a tumor suppressor and inhibits the growth and metastases of GAC cells through YAP1 downregulation [88]. A drug verteporfin impaired YAP1 and TEAD interaction to suppress the expression of downstream targets in pancreatic cancer [89]. Figure 2B illustrates the interplay among Hippo/YAP1, other oncogenes and the immunosuppressive microenvironment.

\subsubsection{Hippo/YAP1 and Cancer Stem Cells}

ALDH1 is a CSC marker in many different tumor tissues. Inducible YAP1 expression in esophageal cancer cells increased ALDH1+ cells, double (ALDH1+/CD44+) positive cells, and greatly increased tumor-sphere numbers and size; conversely, YAP1 knockdown decreased ALDH1+ cells and double (ALDH1+/CD44+) positive cells, leading to significant reduction of tumor-sphere size and number, which indicated that YAP1 in esophageal cancer cells endowed tumor cells with CSC properties [83]. YAP1 was highly upregulated in peritoneal carcinomatosis (PC cells or malignant ascites), conferred CSC properties and appeared to be a metastatic driver. YAP1 expression significantly correlates with 
CSC genes such as Sox9, Hes1, Prom1 (CD133) and Itga6 (CD49f) [84]. Sox9 is a CSC marker in GI tract and a YAP1 target gene that controls CSC features in esophageal cancer [83]. Hes1, a Notch signaling target, has been reported to regulate CSC features in the GI tract. CD133 and CD49f are reported CSC markers in many tumor types. YAP1 expression is correlated with the proportion of ALDH1+ cells in PC specimens. CyTOF (mass cytometry by time of flight) analyses further revealed that YAP1 expression is highly associated with ALDH1, Hes1, CD133, and CD49f suggesting that high expression of YAP1 correlates with a CSC signature in PC [84].

\subsubsection{Interplay Between Hippo/YAP1 and the Immunosuppressive Stroma}

An innate antiviral immunity gene, the interferon regulatory transcription factor 3 (IRF3) is essential for innate immunity against viral infection and cancer, its expression is positively correlated with YAP1 and its target genes in GAC. IRF3 interacts with both YAP1 and TEAD4 by cobinding in the nucleus to enhance their interaction, promoting nuclear translocation, and activation of YAP1. Knockdown or pharmacological targeting of IRF3 by amlexanox, a drug used clinically for anti-inflammatory treatment, inhibits GAC growth in a YAP-dependent manner. Therefore, IRF3 is identified as a positive regulator for YAP1 [90] Shibata et al. (2018) presented review of the Hippo/YAP1 pathway on tumorigenesis and immunosuppression in TME [79]. YAP1 and its paralog TAZ act as transcriptional coactivators with TEADs to mediate this pathway. YAP1 in cancer cells upregulates cytokines and chemokines TNF $\alpha$, IL6, CSF1-3, CXCL5, CCL2, CSF1 that are associated with recruitment of $\mathrm{M} 2 \mathrm{M} \phi$, Tregs and MDSCs (myeloid-derived suppressor cells) and inhibition of NK cells [79]. Tregs suppress antitumor immunity and can be induced by malignant cells [91].

A study reported that YAP1 is essential for Treg-mediated suppression of antitumor immunity. FOXP3 is a transcription factor expressed in Tregs that is required for its function. It was discovered that YAP1 upregulates activin signaling, and amplifies TGF- $\beta$ /SMAD activation, bolsters FOXP3 expression and Treg function. YAP1 deficiency resulted in dysfunctional Tregs unable to suppress antitumor immunity or promote tumor growth in mice. Chemical YAP1 antagonism and knockout or blockade of the YAP1-regulated activin receptor similarly improved antitumor immunity. It was concluded that YAP1 as an unexpected amplifier of Treg-reinforcing pathway with significant potential being an anticancer immunotherapeutic target [85].

Another study revealed that YAP1 displays nuclear translocation and works with TEAD to activate transcription of the crucial inflammatory cytokine IL-1 $\beta$ in GACs infected with $H$. pylori. As IL-1 $\beta$ accounts for inflammation-associated tumorigenesis, this process leads to gastric carcinogenesis. YAP1 plays a major role in inflammation amplification by activating inflammatory cytokine genes, YAP1's coactivator TEAD binds to IL-1 $\beta$ promoter thus directly regulates IL-1 $\beta$ transcription. Deleting IL-1 $\beta$ by siRNA partially neutralized YAP's ability to promote proliferation [92]. It has been identified hyperactivated Hippo/YAP1 drives CXCL5 upregulation in cancer cells through YAP/TEAD complex and promoting MDSC recruitment, and the latter is via heterotypic CXCL5-CXCR2 signaling [93]. Studies in glioblastoma multiforme (GBM) models established that PTEN deficiency activates YAP1, which directly upregulates lysyl oxidase (LOX) expression. LOX functions as a potent macrophage chemoattractant. These infiltrating macrophages secrete SPP1 and then sustains glioma cell survival and stimulates angiogenesis. This symbiotic glioma-macrophage interplay provides potential therapeutic targets [94]. 


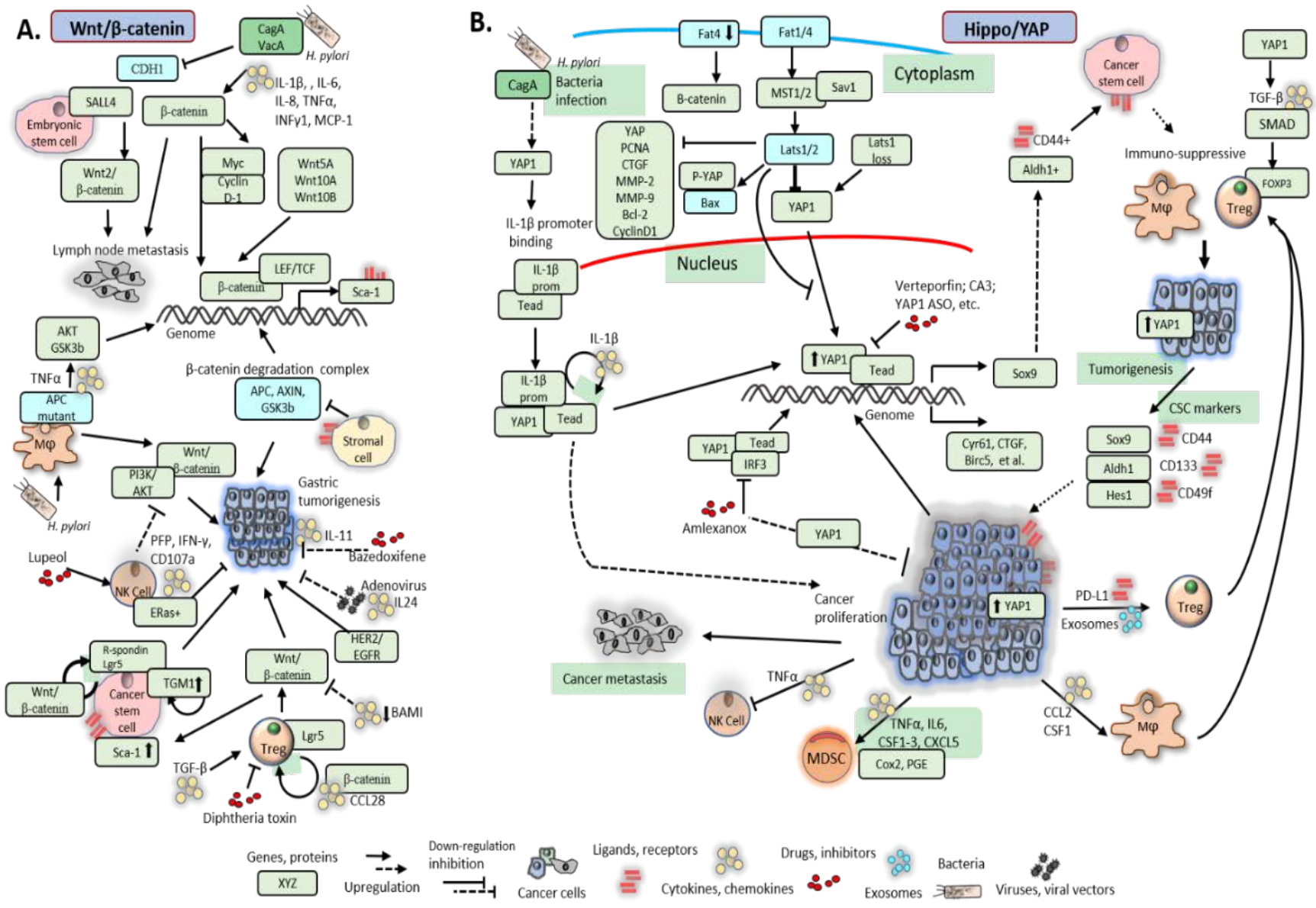

Figure 2 Interplay between oncogenes and the tumor-immune interactions mediated by Wnt/ $\beta$-catenin and Hippo/YAP1 signaling. A. Interplay mediated by $W n t / \beta$-catenin signaling. Bacterial CagA/VacA turn on $\beta$-catenin and suppress $\mathrm{CDH}-1$, driving $\beta$ catenin/LEF/TCF axis signals, like Wnt5A/10A/10B turned on $\beta$-catenin. M2 M $\phi$ secretes TNF $\alpha$ and with help of mutated APC turns on Wnt/ $\beta$-catenin, resulting in neoplastic epithelial cells. Second, cancer stem cell associated SALL4 acts through $W n t / \beta$-catenin to cause lymph node metastasis. Cancer stem cell associated TGM1 and activated Sca1 promote gastric tumor self-renewal and proliferation. Ligand Lgr5 in Treg cells secreted CCL28 promoted Treg cells self-renewal and Wnt/ $\beta$-catenin activation. TGF- $\beta 1$ and chemokine CCL28 works with Tregs to promote cancer stem marker Lgr5. Notably tumor stromal cells block $\beta$-catenin degradation complex that increases $\beta$-catenin and promotes gastric tumorigenesis. B. Crosstalk of Hippo/YAP pathway and TME. Tumor suppressors FAT1/4 and LATS1/2 keeps delicate balance in suppressing YAP1 activation. YAP1 binds co-transactivators Tead1/2/3/4 to turn on some oncogenic genes, Sox6, Cyr61, CTGF, Birc5 etc., some of which act as stem cell markers, such as Sox9, ALDH1 and CD44, which recruit tumor cells-associated M2 M $\phi$ and Tregs to amplify those CSC markers. Tumor secretes cytokines CCL2 and CSF1 to stimulate M2 M $\phi$, that autocrine feedback augments the process of tumorigenesis and cancer proliferation. Again $\mathrm{H}$. pylori's CagA induces YAP1, and Tead binds IL-1 $\beta$ promoter, which turns on IL-1 $\beta$ transcription in a positive feedback, exacerbating gastric cancerogenesis. 


\section{Conclusions}

Cancers in multicellular organisms are believed to be caused by accumulated genetic alterations with activated oncogenes and/or inactivated tumor suppressor genes. Mutated or highly expressed oncogenes derived from normal functional proto-oncogenes are literally the cancer-causing genes. Oncogenes are activated either by viruses or bacteria or other environmental factors, epigenetics and cross-talks with other oncogenic pathways and interplay with cancer stroma to play immunosuppressive functions to further facilitate tumor growth and metastases. In this review, we illustrated the key oncogenes including Ras/Myc, EGFR/HER2, PI3K/mTOR, Wnt/ $\beta$-catenin and Hippo/YAP1 focusing on their interactions with each other and their immunosuppressive stroma to promote tumorigenesis, proliferation and cancer metastases, focusing on GAC. New therapeutic strategies should target both oncogenes and the immune suppressive stroma activated as mentioned in this review.

\section{Abbreviations}

CSC: cancer stem cell; EMT: epithelial-mesenchymal transition; FDA: U.S. Food and Drug Administration; GC: gastric cancer; GAC: gastric adenocarcinoma; GEC: gastroesophageal cancer; GI: gastrointestinal; HGFR: hepatocyte growth factor receptor; IHC: immunohistochemistry; IL: interleukin; IncRNA: long noncoding RNA; MALT: mucosa-associated lymphoid tissue lymphoma; MDSC: myeloid-derived suppressor cells; MSI: Microsatellite instability; NK cell: natural killer cell; PC: peritoneal carcinomatosis; TME: tumor microenvironment; Treg: regulatory T-cell; NRF2: nuclear factor E2-related factor 2; LEDGF: transcriptional co-activator lens epithelium-derived growth factor; TAM: Tumor associated macrophage.

\section{Author Contributions}

Conception and design: J Jin, S. Song, J Ajani; Manuscript writing: J Jin, S. Song, J Ajani; Revision and final approval of manuscript: J Jin, S. Song, J Ajani. All authors read and approved the final manuscript.

\section{Funding}

This work was supported by an MD Anderson Institutional Research Grant (3-0026317 to S. Song); and grants from Department of Defense (CA160433 and CA170906 to S. Song and CA160445 to J Ajani); and the National Institutes of Health (CA129906, CA138671, and CA172741 to J.A. Ajani). Supported in part by the Caporella family, the Park family, the Dallas family, the Dio family, the Frankel family, the Smith family, Anonymous donor, the McNeil family, the Stupid Strong Foundation (Dallas, TX), and the Gastric Cancer Foundation (San Francisco, CA).

\section{Competing Interests}

No potential conflicts of interest were disclosed by all authors. 


\section{References}

1. Torry DS, Cooper GM. Proto-oncogenes in development and cancer. Am J Reprod Immunol. 1991; 25: 129-132.

2. Cai QL, Yuan ZH, Ke L. Infectious agents associated cancers: Epidemiology and molecular biology. New York: Springer Berlin Heidelberg; 2017.

3. Elimova E, Song S, Shimodaira Y, Lin Q, Ajani JA. Exploiting molecular and immune biology of gastric and gastroesophageal adenocarcinomas to discover novel therapeutic targets. Ann Surg Oncol. 2016; 23: 3786-3791.

4. Weiss RA, Vogt PK. 100 years of rous sarcoma virus. J Exp Med. 2011; 208: 2351-2355.

5. Ajani JA, Lee J, Sano T, Janjigian YY, Fan D, Song S. Gastric adenocarcinoma. Nat Rev Dis Primers. 2017; 3: 17036. doi: 10.1038/nrdp.2017.36.

6. Molaei F, Forghanifard MM, Fahim Y, Abbaszadegan MR. Molecular signaling in tumorigenesis of gastric cancer. Iran Biomed J. 2018; 22: 217-230.

7. Zhao J, Liang Q, Cheung KF, Kang W, Dong Y, Lung RW, et al. Somatostatin receptor 1 , a novel EBV-associated CpG hypermethylated gene, contributes to the pathogenesis of EBV-associated gastric cancer. Br J Cancer. 2013; 108: 2557-2564.

8. Szkaradkiewicz A, Karpiński TM, Majewski J, Malinowska K, Goślińska-Kuźniarek O, Linke K. The participation of $\mathrm{p} 53$ and bcl-2 proteins in gastric carcinomas associated with helicobacterpylori and/or Epstein-Barr Virus (EBV). Pol J Microbiol. 2015; 64: 211-216.

9. Wang J, Zheng X, Qin Z, Wei L, Lu Y, Peng Q, et al. Epstein-Barr virus miR-BART3-3p promotes tumorigenesis by regulating the senescence pathway in gastric cancer. J Biol Chem. 2019; 294: 4854-4866.

10. Li S, Bai L, Dong J, Sun R, Lan K. Kaposi's sarcoma-associated herpesvirus: Epidemiology and molecular biology. Adv Exp Med Bio. 2017; 1018: 91-127.

11. Cai Q, Yuan Z. Overview of infectious causes of human cancers. Adv Exp Med Bio. 2017; 1018: 1-9.

12. Pei $Y$, Lewis AE, Robertson ES. Current progress in EBV-Associated B-Cell lymphomas. Adv Exp Med Bio. 2017; 1018: 57-74.

13. Zhang J, Jia L, Tsang CM, Tsao SW. EBV infection and glucose metabolism in nasopharyngeal carcinoma. Adv Exp Med Bio. 2017; 1018: 75-90.

14. Xie Y, Hepatitis B. Virus-associated hepatocellular carcinoma. Adv Exp Med Bio. 2017; 1018: 1121.

15. Sun SC, Elwood J, Béraud C, Greene WC. Human T-cell leukemia virus type I Tax activation of NF-kappa B/Rel involves phosphorylation and degradation of I kappa B alpha and RelA (p65)mediated induction of the c-rel gene. Mol Cell Biol. 1994; 14: 7377-7384.

16. Schafer KA. The cell cycle: A review. Vet Pathol. 1998; 35: 461-478.

17. Engelman AN, Singh PK. Cellular and molecular mechanisms of HIV-1 integration targeting. Cell Mol Life Sci. 2018; 75: 2491-2507.

18. Atkin SJ, Griffin BE, Dilworth SM. Polyoma virus and simian virus 40 as cancer models: History and perspectives. Semin Cancer Biol. 2009; 19: 211-217.

19. Zhu C, Wang Y, Cai C, Cai Q. Bacterial infection and associated cancers. Adv Exp Med Bio. 2017; 1018: 181-191. 
20. Cao S, Shen D, Wang Y, Li L, Zhou L, Wang Y. Potential malignant transformation in the gastric mucosa of immunodeficient mice with persistent mycoplasma penetrans infection. PLoS One. 2017; 12: e0180514. doi: 10.1371/journal.pone.0180514.

21. Wang F, Meng W, Wang B, Qiao L. Helicobacter pylori-induced gastric inflammation and gastric cancer. Cancer Lett. 2014; 345: 196-202.

22. Yousefi B, Mohammadlou M, Abdollahi M, Salek Farrokhi A, Karbalaei M, Keikha M, et al. Epigenetic changes in gastric cancer induction by Helicobacter pylori. J Cell Physiol. 2019; 234: 21770-21784.

23. Varmus H, Pao W, Politi K, Podsypanina K, Du YC. Oncogenes come of age. Cold Spring Harbor Symp Quant Biol. 2005; 70: 1-9.

24. Shih $C$, Weinberg RA. Isolation of a transforming sequence from a human bladder carcinoma cell line. Cell. 1982; 29: 161-169.

25. Venkateswaran N, Conacci-Sorrell M. MYC leads the way. Small GTPases. 2020; 11: 86-94.

26. Yao X, Ajani JA, Song S. Molecular biology and immunology of gastric cancer peritoneal metastasis. Transl Gastroenterol Hepatol. 2020; 5: 57. doi: 10.21037/tgh.2020.02.08.

27. Waldmann TA. Cytokines in cancer immunotherapy. Cold Spring Harb Perspect Biol. 2018; 10 : a028472. doi: 10.1101/cshperspect.a028472.

28. Nagarsheth $\mathrm{N}$, Wicha MS, Zou W. Chemokines in the cancer microenvironment and their relevance in cancer immunotherapy. Nat Rev Immunol. 2017; 17: 559-572.

29. Cicenas J, Kvederaviciute K, Meskinyte I, Meskinyte-Kausiliene E, Skeberdyte A, Cicenas J. KRAS, TP53, CDKN2A, SMAD4, BRCA1, and BRCA2 mutations in pancreatic cancer. Cancers. 2017; 9; 42-48.

30. Kamisawa T, Wood LD, Itoi T, Takaori K. Pancreatic cancer. Lancet. 2016; 388: 73-85.

31. Zhao W, Chan TL, Chu KM, Chan AS, Stratton MR, Yuen ST, et al. Mutations of BRAF and KRAS in gastric cancer and their association with microsatellite instability. Int J Cencer. 2004; 108: 167-169.

32. Dang CV. MYC on the path to cancer. Cell. 2012; 149: 22-35.

33. Heindl S, Eggenstein E, Keller S, Kneissl J, Keller G, Mutze K, et al. Relevance of MET activation and genetic alterations of KRAS and E-cadherin for cetuximab sensitivity of gastric cancer cell lines. J Cancer Res Clin Oncol. 2012; 138: 843-858.

34. Kubota $E$, Kataoka $H$, Tanaka M, Okamoto $Y$, Ebi $M$, Hirata $Y$, et al. ERas enhances resistance to CPT-11 in gastric cancer. Int J Cancer. 2011; 31: 3353-3360.

35. Su CC. Tanshinone IIA inhibits gastric carcinoma AGS cells by decreasing the protein expression of VEGFR and blocking Ras/Raf/MEK/ERK pathway. Int J Mol Med. 2018; 41: 2389-2396.

36. Okumura T, Ericksen RE, Takaishi S, Wang SS, Dubeykovskiy Z, Shibata W, et al. K-ras mutation targeted to gastric tissue progenitor cells results in chronic inflammation, an altered microenvironment, and progression to intraepithelial neoplasia. Cancer Res. 2010; 70: 84358445.

37. Kortlever RM, Sodir NM, Wilson CH, Burkhart DL, Pellegrinet L, Brown Swigart L, et al. Myc cooperates with Ras by programming inflammation and immune suppression. Cell. 2017; 171: 1301-1315.

38. Casey SC, Tong L, Li Y, Do R, Walz S, Fitzgerald KN, et al. MYC regulates the antitumor immune response through CD47 and PD-L1. Science. 2016; 352: 227-231. 
39. Kim YB, Ahn JM, Bae WJ, Sung CO, Lee D. Functional loss of ARID1A is tightly associated with high PD-L1 expression in gastric cancer. Int J Cancer. 2019; 145: 916-926.

40. Arienti C, Pignatta S, Tesei A. Epidermal growth factor receptor family and its role in gastric cancer. Front Oncol. 2019; 9: 1308. doi: 10.3389/fonc.2019.01308.

41. Albarello L, Pecciarini L, Doglioni C. HER2 testing in gastric cancer. Adv Anat pathol. 2011; 18 : 53-59.

42. Aznab M, Maleksabet D, Khazaei S, Khazaei M, Rezaei M. The role of human Epidermal Growth Factor Receptor (HER2/neu) in the prognosis of patients with gastric cancer. Asian Pac J Cancer Prev. 2019; 20: 1989-1994.

43. Tomioka H, Mukohara T, Kataoka Y, Ekyalongo RC, Funakoshi $Y$, Imai $Y$, et al. Inhibition of the mTOR/S6K signal is necessary to enhance fluorouracil-induced apoptosis in gastric cancer cells with HER2 amplification. Int J Oncol. 2012; 41: 551-558.

44. Bai M, Song N, Che X, Wang X, Qu X, Liu Y. Chk1 activation attenuates sensitivity of lapatinib in HER2-positive gastric cancer. Cell Biol Int. 2018; 42: 781-793.

45. Maron SB, Catenacci DV. Novel targeted therapies for esophagogastric cancer. Surg Oncol Clin N Am. 2017; 26: 293-312.

46. Wang M, Ferreira RB, Law ME, Davis BJ, Yaaghubi E, Ghilardi AF, et al. A novel proteotoxic combination therapy for EGFR+ and HER2+ cancers. Oncogene. 2019; 38: 4264-4282.

47. Gao L, Wang L, Dai T, Jin K, Zhang Z, Wang S, et al. Tumor-derived exosomes antagonize innate antiviral immunity. Nat Immunol. 2018; 19: 233-245.

48. Karan D, Holzbeierlein J, Thrasher JB. Macrophage inhibitory cytokine-1: Possible bridge molecule of inflammation and prostate cancer. Cancer Res. 2009; 69: 2-5.

49. Kim KK, Lee JJ, Yang Y, You KH, Lee JH. Macrophage inhibitory cytokine-1 activates AKT and ERK$1 / 2$ via the transactivation of ErbB2 in human breast and gastric cancer cells. Carcinogenesis. 2008; 29: 704-712.

50. Tsao LC, Crosby EJ, Trotter TN, Agarwal P, Hwang BJ, Acharya C, et al. CD47 blockade augmentation of trastuzumab antitumor efficacy dependent on antibody-dependent cellular phagocytosis. JCl Insight. 2019; 4; e131882. doi: 10.1172/jci.insight.131882.

51. Su S, Zhao J, Xing Y, Zhang X, Liu J, Ouyang Q, et al. Immune checkpoint inhibition overcomes ADCP-Induced immunosuppression by macrophages. Cell. 2018; 175: 442-457.

52. Monypenny J, Milewicz H, Flores-Borja F, Weitsman G, Cheung A, Chowdhury R, et al. ALIX regulates tumor-mediated immunosuppression by controlling egfr activity and PD-L1 presentation. Cell Rep. 2018; 24: 630-641.

53. Bilgin B, Sendur MA, Bülent Akıncı M, Şener Dede D, Yalçın B. Targeting the PD-1 pathway: A new hope for gastrointestinal cancers. Curr Med Res Opin. 2017; 33: 749-759.

54. Freitag H, Christen F, Lewens F, Grass I, Briest F, Iwaszkiewicz S, et al. Inhibition of mTOR's catalytic site by PKI-587 is a promising therapeutic option for gastroenteropancreatic neuroendocrine tumor disease. Neuroendocrinology. 2017; 105: 90-104.

55. Lang SA, Hackl C, Moser C, Fichtner-Feigl S, Koehl GE, Schlitt HJ, et al. Implication of RICTOR in the mTOR inhibitor-mediated induction of insulin-like growth factor-I receptor (IGF-IR) and human epidermal growth factor receptor-2 (Her2) expression in gastrointestinal cancer cells. Biochim Biophys Acta. 2010; 1803: 435-442. 
56. Kim ST, Kim SY, Klempner SJ, Yoon J, Kim N, Ahn S, et al. Rapamycin-insensitive companion of mTOR (RICTOR) amplification defines a subset of advanced gastric cancer and is sensitive to AZD2014-mediated mTORC1/2 inhibition. Ann Oncol. 2017; 28: 547-554.

57. Lang SA, Hackl C, Moser C, Fichtner-Feigl S, Koehl GE, Schlitt HJ, et al. Implication of RICTOR in the mTOR inhibitor-mediated induction of insulin-like growth factor-I receptor (IGF-IR) and human epidermal growth factor receptor-2 (Her2) expression in gastrointestinal cancer cells. Biochimica Et Biophysica Acta. 2010; 1803: 435-442.

58. Wu H, Han Y, Rodriguez Sillke Y, Deng H, Siddiqui S, Treese C, et al. Lipid droplet-dependent fatty acid metabolism controls the immune suppressive phenotype of tumor-associated macrophages. EMBO Mol Med. 2019; 11: e10698. doi: 10.15252/emmm.201910698.

59. Sun L, Wang $Q$, Chen B, Zhao $Y$, Shen B, Wang $H$, et al. Gastric cancer mesenchymal stem cells derived IL-8 induces PD-L1 expression in gastric cancer cells via STAT3/mTOR-C-Myc signal axis. Cell Death Dis. 2018; 9: 928. doi: 10.1038/s41419-018-0988-9.

60. Koizumi K, Kato S, Sakurai H, Hashimoto I, Yasumoto K, Saiki I. Therapeutics target of CXCR4 and its downstream in peritoneal carcinomatosis of gastric cancer. Front Biosci. 2012; 4: 269-276.

61. Chen G, Chen SM, Wang X, Ding XF, Ding J, Meng LH. Inhibition of chemokine (CXC motif) ligand 12/chemokine (CXC motif) receptor 4 axis (CXCL12/CXCR4)-mediated cell migration by targeting mammalian target of rapamycin (mTOR) pathway in human gastric carcinoma cells. J Biol Chem. 2012; 287: 12132-12141.

62. Daniel SK, Seo YD, Pillarisetty VG. The CXCL12-CXCR4/CXCR7 axis as a mechanism of immune resistance in gastrointestinal malignancies. Semin Cancer Biol. 2020; 65: 176-188.

63. Thiem S, Pierce TP, Palmieri M, Putoczki TL, Buchert $M$, Preaudet $A$, et al. mTORC1 inhibition restricts inflammation-associated gastrointestinal tumorigenesis in mice. J Clin Invest. 2013; 123: 767-781.

64. Shang $S$, Hua F, Hu ZW. The regulation of $\beta$-catenin activity and function in cancer: Therapeutic opportunities. Oncotarget. 2017; 8: 33972-33989.

65. Oguma K, Oshima H, Aoki M, Uchio R, Naka K, Nakamura S, et al. Activated macrophages promote Wnt signalling through tumour necrosis factor-alpha in gastric tumour cells. Embo J. 2008; 27: 1671-1681.

66. DeNardo DG, Johansson M, Coussens LM. Inflaming gastrointestinal oncogenic programming. Cancer Cell. 2008; 14: 7-9.

67. Wu XT, Liu JQ, Lu XT, Chen FX, Zhou ZH, Wang T, et al. The enhanced effect of lupeol on the destruction of gastric cancer cells by NK cells. Int Immunopharmacol. 2013; 16: 332-340.

68. Ji L, Qian W, Gui L, Ji Z, Yin P, Lin GN, et al. Blockade of $\beta$-catenin-induced CCL28 suppresses gastric cancer progression via inhibition of treg cell infiltration. Cancer Res. 2020; 80: 2004-2016.

69. Kirikoshi H, Sekihara H, Katoh M. Up-regulation of WNT10A by tumor necrosis factor alpha and Helicobacter pylori in gastric cancer. Int J Oncol. 2001; 19: 533-536.

70. Saitoh T, Kirikoshi H, Mine T, Katoh M. Proto-oncogene WNT10B is up-regulated by tumor necrosis factor alpha in human gastric cancer cell line MKN45. Int J Oncol. 2001; 19: 1187-1192.

71. Saitoh T, Katoh M. Expression and regulation of WNT5A and WNT5B in human cancer: Upregulation of WNT5A by TNFalpha in MKN45 cells and up-regulation of WNT5B by beta-estradiol in MCF-7 cells. Int J Mol Med. 2002; 10: 345-349. 
72. Thilakasiri P, Huynh J, Poh AR, Tan CW, Nero TL, Tran K, et al. Repurposing the selective estrogen receptor modulator bazedoxifene to suppress gastrointestinal cancer growth. EMBO Mol Med. 2019; 11: e9539. doi: 10.15252/emmm.201809539.

73. Liu X, Qian Q, Xu P, Wolf F, Zhang J, Zhang D, et al. A novel conditionally replicating "armed" adenovirus selectively targeting gastrointestinal tumors with aberrant wnt signaling. Hum Gene Ther. 2011; 22: 427-437.

74. Liu K, Song X, Ma H, Liu L, Wen X, Yu J, et al. Knockdown of BAMBI inhibits $\beta$-catenin and transforming growth factor $\beta$ to suppress metastasis of gastric cancer cells. Mol Med Rep. 2014; 10: $874-880$.

75. Liu XS, Lin XK, Mei Y, Ahmad S, Yan CX, Jin HL, et al. Regulatory T cells promote overexpression of Lgr5 on gastric cancer cells via TGF-beta1 and confer poor prognosis in gastric cancer. Front Immunol. 2019; 10: 1741. doi: 10.3389/fimmu.2019.01741.

76. Huang $\mathrm{H}, \mathrm{Chen} \mathrm{Z}$, Ni X. Tissue transglutaminase-1 promotes stemness and chemoresistance in gastric cancer cells by regulating Wnt/ $\beta$-catenin signaling. Exp Biol Med. 2017; 242: 194-202.

77. Park JW, Park JM, Park DM, Kim DY, Kim HK. Stem cells Antigen-1 Enriches for a cancer Stem Cell-Like subpopulation in mouse gastric cancer. Stem Cells. 2016; 34: 1177-1187.

78. Shamai Y, Alperovich DC, Yakhini Z, Skorecki K, Tzukerman M. Reciprocal reprogramming of cancer cells and associated mesenchymal stem cells in gastric cancer. Stem Cells. 2019; 37: 176189.

79. Shibata M, Ham K, Hoque MO. A time for YAP1: Tumorigenesis, immunosuppression and targeted therapy. Int J Cancer. 2018; 143: 2133-2144.

80. Li F, Xu Y, Liu B, Singh PK, Zhao W, Jin J, et al. YAP1-mediated CDK6 activation confers radiation resistance in esophageal cancer - rationale for the combination of YAP1 and CDK4/6 inhibitors in esophageal cancer. Clin Cancer Res. 2019; 25: 2264-2277.

81. Song S, Xie M, Scott AW, Jin J, Ma L, Dong X, et al. A novel YAP1 inhibitor targets CSC-enriched Radiation-Resistant cells and exerts strong antitumor activity in esophageal adenocarcinoma. Mol Cancer Ther. 2018; 17: 443-454.

82. Song S, Honjo S, Jin J, Chang SS, Scott AW, Chen Q, et al. The hippo coactivator YAP1 mediates EGFR overexpression and confers chemoresistance in esophageal cancer. Clin Cancer Res. 2015; 21: 2580-2590.

83. Song S, Ajani JA, Honjo S, Maru DM, Chen $Q$, Scott AW, et al. Hippo coactivator YAP1 upregulates SOX9 and endows esophageal cancer cells with stem-like properties. Cancer Res. 2014; 74: 4170-4182.

84. Ajani JA, Xu Y, Huo L, Wang R, Li Y, Wang Y, et al. YAP1 mediates gastric adenocarcinoma peritoneal metastases that are attenuated by YAP1 inhibition. Gut. 2020; 2019: 319748. doi: 10.1136/gutjnl-2019-319748.

85. Ni X, Tao J, Barbi J, Chen Q, Park BV, Li Z, et al. YAP is essential for treg-mediated suppression of antitumor immunity. Cancer Disc. 2018; 8: 1026-1043.

86. Katoh M, Katoh M. Transcriptional mechanisms of WNT5A based on NF-kappaB, Hedgehog, TGFbeta, and Notch signaling cascades. Int J Mol Med. 2009; 23: 763-769.

87. Ma L, Cui J, Xi H, Bian S, Wei B, Chen L. Fat4 suppression induces Yap translocation accounting for the promoted proliferation and migration of gastric cancer cells. Cancer Biol Ther. 2016; 17: 36-47. 
88. Zhang J, Wang G, Chu SJ, Zhu JS, Zhang R, Lu WW, et al. Loss of large tumor suppressor 1 promotes growth and metastasis of gastric cancer cells through upregulation of the YAP signaling. Oncotarget. 2016; 7: 16180-16193.

89. Wei H, Wang F, Wang Y, Li T, Xiu P, Zhong J, et al. Verteporfin suppresses cell survival, angiogenesis and vasculogenic mimicry of pancreatic ductal adenocarcinoma via disrupting the YAP-TEAD complex. Cancer sci. 2017; 108: 478-487.

90. Jiao S, Guan J, Chen M, Wang W, Li C, Wang Y, et al. Targeting IRF3 as a YAP agonist therapy against gastric cancer. J Exp Med. 2018; 215: 699-718.

91. Ansell SM, Vonderheide RH. Cellular composition of the tumor microenvironment. Am Soc Clin Oncol Educ Book. 2013. doi: 10.1200/EdBook_AM.2013.33.e91.

92. Wu Y, Shen L, Liang X, Li S, Ma L, Zheng L, et al. Helicobacter pylori-induced YAP1 nuclear translocation promotes gastric carcinogenesis by enhancing IL-1 $\beta$ expression. Cancer Med. 2019; 8: 3965-3980.

93. Wang G, Lu X, Dey P, Deng P, Wu CC, Jiang S, et al. Targeting YAP-dependent MDSC infiltration impairs tumor progression. Cancer Disc. 2016; 6: 80-95.

94. Chen $P$, Zhao D, Li J, Liang X, Li J, Chang A, et al. Symbiotic macrophage-glioma cell interactions reveal synthetic lethality in pten-null glioma. Cancer Cell. 2019; 35: 868-884.

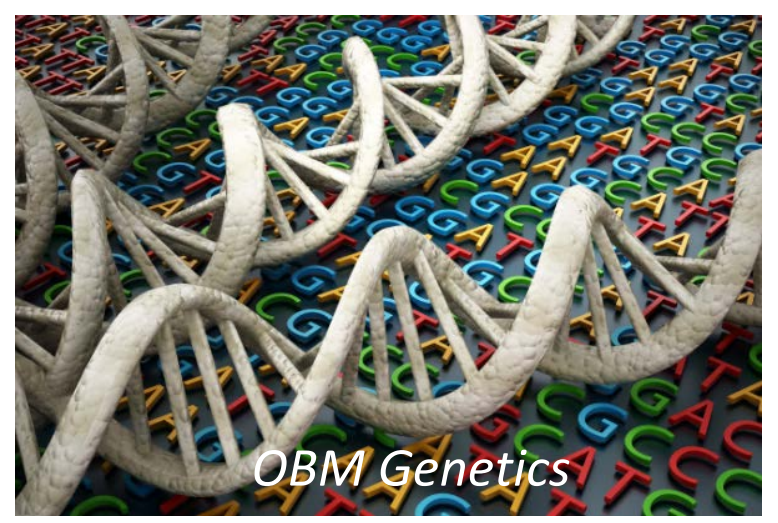

Enjoy OBM Genetics by:

1. Submitting a manuscript

2. Joining in volunteer reviewer bank

3. Joining Editorial Board

4. Guest editing a special issue

For more details, please visit: http://www.lidsen.com/journals/genetics 